(6) OPEN ACCESS

- Additional material is published online only. To view please visit the journal online (http://dx.doi.org/10.1136/ practneurol-2014-000989).

${ }^{1}$ Institute of Clinical Neurosciences, Royal Prince Alfred Hospital, Camperdown, New South Wales, Australia ${ }^{2}$ Departments of Medicine and Physiology, Yong Loo Lin School of Medicine, National University of Singapore, \#09-01 Neuroscience Research Centre, Centre for Translational Medicine, Singapore, Singapore ${ }^{3}$ Brain \& Mind Research Institute, University of Sydney, Camperdown, New South Wales, Australia

\section{Correspondence to} Professor Matthew C Kiernan, Brain \& Mind Research Institute, The University of Sydney, Room 438, Level 4, 94 Mallett Street Camperdown, NSW 2050,

Australia;

matthew.kiernan@sydney.edu.au

Accepted 13 October 2014 Published Online First 29 October 2014

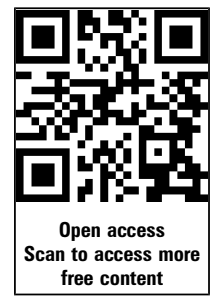

CrossMark

To cite: Carswell $C$,

Northey LC, Davies L, et al.

Pract Neurol 2015;15:76-79.

\title{
Progressive bilateral facial weakness
}

\author{
Christopher Carswell, ${ }^{1}$ Luke C Northey, ${ }^{1}$ Leo Davies, ${ }^{1}$ Nobuhiro Yuki, ${ }^{2}$ \\ Matthew C Kiernan ${ }^{1,3}$
}

A 72-year-old man presented with sudden onset of right-sided facial muscle weakness and slurred speech and a 3-day history of fevers, myalgia and sore throat. There was no hyperacusis, change in taste or lacrimation. His history included untreated hepatitis $\mathrm{C}$ diagnosed 10 years before, but quiescent at his annual liver review 8 weeks before presentation. $\mathrm{He}$ smoked cannabis intermittently but there was no recent alcohol or other drug abuse. There was a history of promiscuity with female partners.

On examination, he was alert and orientated. His blood pressure was elevated at $159 / 86 \mathrm{~mm} \mathrm{Hg}$ but other vital signs and general examination were normal. Neurological examination showed a severe right-sided lower motor facial weakness with Bell's phenomenon (see online supplementary video). The remaining cranial nerve examination was normal, including eye movements, pupillary responses, facial sensation, hearing, taste and otoscopy. There was mild left hip flexion weakness but preserved deep tendon reflexes. There was reduced sensation to temperature in a glove-and-stocking pattern; joint-position sense, light touch and pain sensation were intact. There was mild gait instability on walking tandem.

His condition progressed rapidly and on day 2 of admission he developed left-sided facial weakness (figure 1) with bilateral, proximal lower limb weakness. His paraesthesias were more painful. His lower limb deep tendon reflexes were no longer present (see online supplementary video). Spirometry remained normal. He was transferred to a high dependency unit for ongoing monitoring.

\section{Question 1}

What is the differential diagnosis and what are the most appropriate investigations?

\section{COMMENT}

Lower motor neurone facial weakness and (to a lesser extent) the absence of deep tendon reflexes are highly localising signs. The lesion explaining the facial and the limb weakness can be localised to somewhere between the peripheral nerve roots and muscles; however, there are many possible causes (table 1). Dominant sensory symptoms (paraesthesias) make either a neuromuscular junction pathology (eg, botulism or myasthenia gravis) or a myopathy (eg, polymyositis) less likely. The bilateral, although asynchronous, onset of the facial weakness suggests that generalised or multifocal disease rather than a solitary lesion. The progression over days favours an infective or inflammatory/immune cause and makes a vascular, neoplastic or degenerative process less likely.

Other possibilities include listerial rhombencephalitis, tuberculosis, sarcoidosis, Sjögren's syndrome or other causes of multiple mononeuropathy, such as vasculitis, HIV, syphilis or Lyme disease. The patient had not travelled outside Australia, making Lyme disease unlikely. 'Facial-onset sensorimotor neuropathy', a novel neurodegenerative neuropathy, is also possible; however, the clinical course is usually indolent and progresses to involve other limbs. ${ }^{1}$ A postinfective immune phenomenon would be an alternative.

Normal or negative investigations included HIV, serum ACE, creatine 


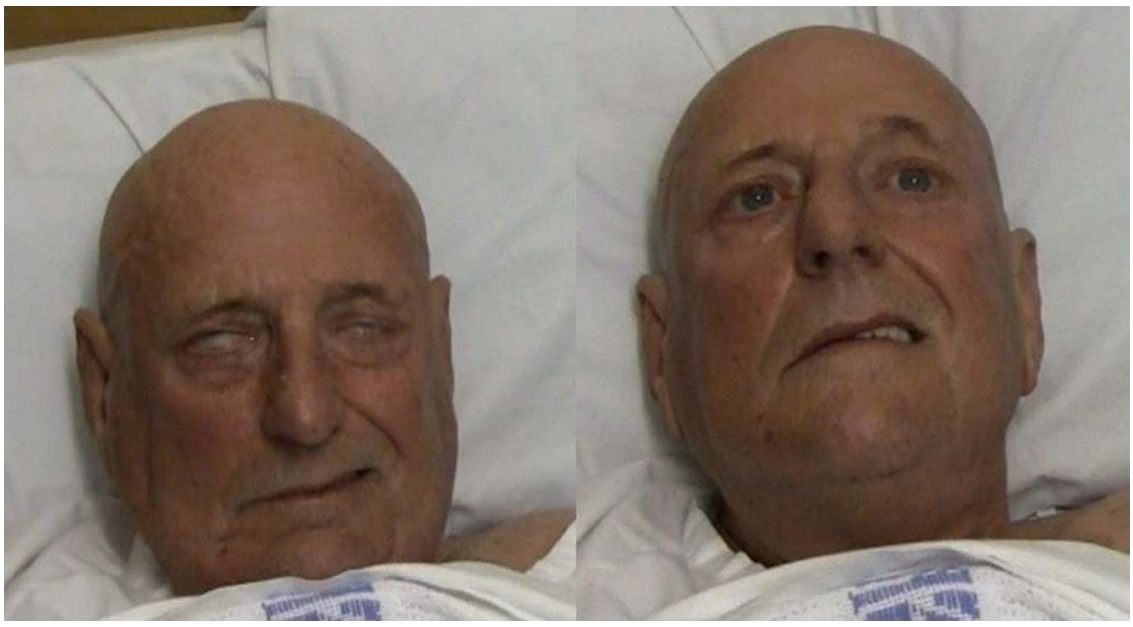

Figure 1 Bifacial lower motor neurone weakness (see online supplementary video).

Table 1 The differential diagnosis of acute bifacial weakness

\begin{tabular}{|c|c|}
\hline Causes & Comments \\
\hline Guillain-Barré syndrome & $\begin{array}{l}\text { Presents with a variety of associated features. } \\
\text { Acute inflammatory demyelinating } \\
\text { polyradiculoneuropathy is the most common } \\
\text { subtype to show cranial nerve involvement } \\
\text { and pharyngeal-cervical-brachial weakness } \\
\text { and Miller Fisher syndrome often have facial } \\
\text { weakness. }\end{array}$ \\
\hline
\end{tabular}

Brainstem lesions and malignancy

Lyme disease

Syphilis

Tuberculosis

Sarcoidosis

Sjögren's syndrome

Bell's palsy

'Facial-onset sensorimotor neuropathy'

Neuromuscular disorders

Acute myopathies
Lesions at the level of the pontine tegmentum can affect both facial motor nuclei. These may be meningeal or parenchyma based.

Acute infection may present as Bannwarth syndrome with meningitis, radiculoneuritis and cranial nerve palsies. The facial nerve is most frequently affected, and half of the cases have bilateral facial nerve palsy.

Syphilis displays protean disease manifestations and has the potential to produce cranial neuropathy, including facial nerve palsy, during the tertiary stage of disease with syphilitic meningitis.

Tuberculous meningitis may produce cranial nerve palsies.

Cranial nerve neuropathy, particularly of the facial nerve, is the most common complication of neurosarcoidosis. Bifacial palsy may develop simultaneously or in sequence.

Rare but documented association in the literature

Bilateral Bell's palsy is the most common cause of facial diplegia. It commonly presents with pain around the ear, hyperacusis, loss of taste and lacrimation. However, it should remain as a diagnosis of exclusion.

A novel neurological entity, initially presents with facial sensory deficits, with a slow evolution in a rostral-caudal direction to affect sequentially the neck, upper trunk and limbs.

Botulism or myasthenia gravis commonly present with bifacial weakness.

Polymyositis rarely causes bifacial weakness. kinase, $\mathrm{C}$ reactive protein, antineutrophil cytoplasmic antibody, antinuclear antibody, extractable nuclear antigen, erythrocyte sedimentation rate, blood cultures, antiganglioside antibodies, viral serology and contrast-enhanced MRI of the brain. Cerebrospinal fluid (CSF) examination showed 7 lymphocytes/ $\mu \mathrm{L}$ $(\leq 5)$, protein $1.04 \mathrm{~g} / \mathrm{L}(0.15-0.45 \mathrm{~g} / \mathrm{L})$ and glucose $4.5 \mathrm{mmol} / \mathrm{L} \quad(3.3-4.4 \mathrm{mmol} / \mathrm{L})$. Oligoclonal bands were positive in the serum and CSF. Neurophysiology 1 week after admission showed absent sural nerve sensory potentials, with prolonged distal motor latencies, markedly reduced motor conduction velocities and prolonged and dispersed $\mathrm{F}$ waves bilaterally in the lower limbs, consistent with an acute demyelinating polyneuropathy.

\section{Question 2}

What is the most likely diagnosis?

\section{COMMENT}

In the early stage of Guillain-Barré syndrome, absent or prolonged tibial nerve H-reflex responses are common, paralleling the loss of deep tendon reflexes. This is followed by abnormal F-wave responses, temporal dispersion of distal compound muscle action potentials, prolonged distal motor and F-wave latencies and reduced motor conduction velocity. A-waves and abnormal blink reflexes may also occur early in Guillain-Barré syndrome. ${ }^{2}$ Given that our case had most of these features as well as CSF albuminocytological dissociation, the most likely diagnosis is the 'bifacial weakness with paraesthesias' subtype of Guillain-Barré syndrome. ${ }^{3} 4$

Guillain-Barré syndrome is a postinfective polyneuropathy characterised by progressive limb weakness and areflexia, commonly associated with CSF albuminocytological dissociation and neurophysiological 
evidence of peripheral nerve dysfunction. ${ }^{2}$ GuillainBarré syndrome has several regional variants, ${ }^{3}{ }^{4}$ the most common being Miller Fisher syndrome: this presents with external ophthalmoparesis, ataxia and areflexia, associated with the anti-GQ1b antibodies. ${ }^{2} 4$

In 1994, Allan Ropper described four cases of bifacial weakness with paraesthesias, occurring in a postinfective period with a monophasic illness course and other investigations consistent with Guillain-Barré syndrome. ${ }^{3}$ 'Bifacial weakness with paraesthesias' is now recognised as a localised subtype of Guillain-Barré syndrome, along with a 'pharyngeal-brachial-cervical weakness' and 'paraparetic Guillain-Barré syndrome. ${ }^{4}$

In the largest case series to date, Susuki et al investigated 22 cases of bifacial weakness with paraesthesias and found that $86 \%$ had a preceding illness, with upper respiratory illness more frequent than diarrhoea. There were IgM antibodies to cytomegalovirus in 30\% and to Epstein-Barr virus in 20\% (both significantly more frequently than in Guillain-Barré syndrome). The most common first clinical symptom was distal paraesthesias (86\%); $27 \%$ had a unilateral onset of facial weakness. All cases had CSF albuminocytological dissociation and nerve conduction studies showed motor demyelination in $64 \%$ and sensory abnormalities in $27 \%$. As with previous studies in Guillain-Barré syndrome, anti-GM2 IgM antibodies were more commonly (but not exclusively) present in patients with cytomegalovirus infection. ${ }^{5}$ All of Ropper's 1994 variants now appear in the current classification of Guillain-Barré syndrome. ${ }^{6}$

\section{Question 3}

What is the relationship between antiganglioside antibodies and acute inflammatory neuropathies?

\section{COMMENT}

Gangliosides are glycosphingolipids that are highly expressed in the nervous system. Antibodies against ganglioside antigens sometimes develop with acute inflammatory neuropathies and can help to confirm the diagnosis as well as to distinguish between the clinical subtypes. For example, GQ1b is strongly expressed in the oculomotor, trochlear and abducens nerves, as well as muscle spindles ${ }^{7} ; 83 \%$ of patients with Miller Fisher syndrome have anti-GQ1b antibodies, probably causing the ophthalmoplegia and the cerebellar-like ataxia. ${ }^{7}$ Acute ataxic neuropathies (Guillain-Barré syndrome/Miller Fisher syndrome overlap variants) can be anti-GQ1b or anti-GD1b antibody-positive. In contrast, GT1a is more densely expressed than GQ1b in human glossopharyngeal and vagus nerves: anti-GT1a antibodies are common in the pharyngeal-cervicalbrachial Guillain-Barré syndrome variant (considered a Guillain-Barré syndrome variant rather than Miller

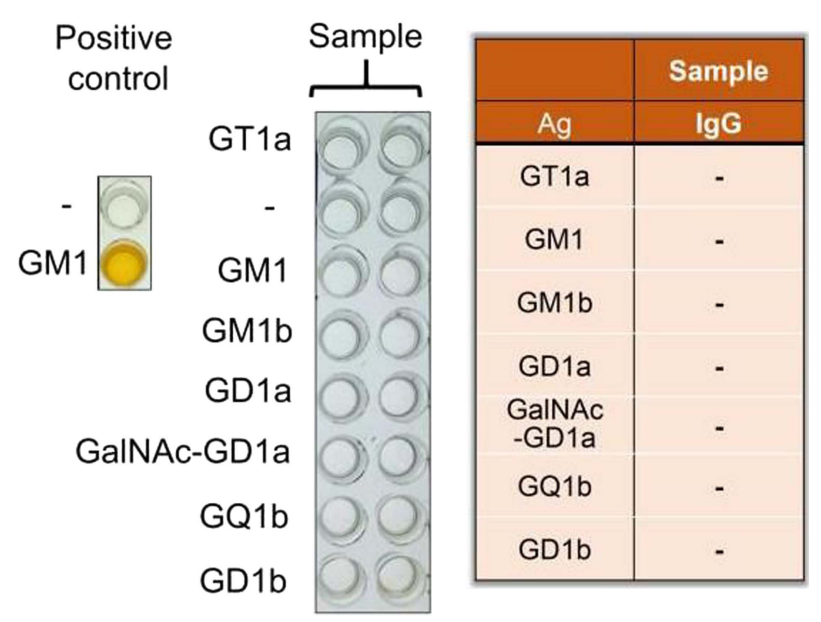

Figure 2 A schematic of antiganglioside antibody screening using ELISA.

Fisher syndrome because limb weakness is a feature). ${ }^{9}$ Anti-GM1 or anti-GD1a antibodies can each occur in acute motor axonal neuropathy, an axonal form of Guillain-Barré syndrome. Paraparetic Guillain-Barré syndrome ${ }^{6}$ is probably a localised subtype of acute motor axonal neuropathy, since anti-GD1a antibodies sometimes occur in this condition. Screening for all ganglioside subtypes helps when investigating atypical causes of weakness (figure 2).

\section{Question 4}

What is the best treatment for 'bifacial weakness with paraesthesias'?

\section{COMMENT}

In Susuki's case series, all patients reached a nadir within 4 weeks and all but one made a good

\section{Practice points}

- Guillain-Barré syndrome is a heterogeneous condition that includes rare regional variants presenting differently from typical Guillain-Barré syndrome; these risks being overlooked in the differential diagnosis.

- Guillain-Barré syndrome can present in localised subtypes, the main ones being 'bifacial weakness with paraesthesias', 'pharyngeal-brachial-cervical weakness' and 'paraparetic Guillain-Barré syndrome'.

- Deep tendon reflexes may be present initially in Guillain-Barré syndrome; patients with weakness of unexplained origin require repeated examination.

- Guillain-Barré syndrome typically gives CSF albuminocytological dissociation and neurophysiological abnormalities but positive antiganglioside antibodies also help with diagnosis. 
recovery. ${ }^{5}$ Some were treated with plasma exchange or intravenous immunoglobulin, although without any firm evidence base. We treated this patient with intravenous immunoglobulin $(2 \mathrm{~g} / \mathrm{kg})$ over 5 days, with regular spirometry, cardiac monitoring and deep vein thromboprophylaxis. Following a second induction course, he regained strength over a month. Two months after the onset of his illness, he is independently mobile with mild facial asymmetry.

Acknowledgements The authors thank Dr Yuki Fukamai at National University of Singapore for performing antiganglioside antibodies. This work was supported by funding to Forefront, a collaborative research initiative from the National Health and Medical Research Council of Australia (Program Grant \#1037746)

Contributors CC, LCN and MK acquired clinical data. CC and MK produced initial drafts. LD performed and interpreted NCS. CC, LD, NY and MK produced the final paper.

Competing interests None.

Patient consent Obtained.

Provenance and peer review Not commissioned; externally peer reviewed. This paper was reviewed by Ben Wakerley, Gloucester, UK.

Open Access This is an Open Access article distributed in accordance with the Creative Commons Attribution Non Commercial (CC BY-NC 4.0) license, which permits others to distribute, remix, adapt, build upon this work noncommercially, and license their derivative works on different terms, provided the original work is properly cited and the use is non-commercial. See: http://creativecommons.org/licenses/by$\mathrm{nc} / 4.0 /$

\section{REFERENCES}

1 Vukic S, Stein TD, Hedley-Whyte ET, et al. FOSMN syndrome: novel insight into disease pathophysiology. Neurology 2012;79:73-9.

2 Vucic S, Kiernan MC, Cornblath DR. Guillain-Barré syndrome: an update. J Clin Neurosci 2009;16:733-41.

3 Ropper A. Further regional variants of acute immune polyneuropathy. Arch Neurol 1994;51:671-5.

4 Wakerley BR, Yuki N. Mimics and chameleons in Guillain-Barré and Miller Fisher syndromes. Pract Neurol 2014. doi: 10.1136/practneurol-2014-000937

5 Susuki K, Koga M, Hirata K, et al. A Guillain Barré variant with prominent facial diplegia. J Neurol 2009;256:1899-905.

6 Wakerley B, Uncini A, Yuki N, the GBS Classification Group; the GBS Classification Group. Guillain-Barré and Miller Fisher syndromes-new diagnostic classification. Nat Rev Neurol 2014;10:537-44.

7 Chiba A, Kusunoki S, Obata H, et al. Serum anti-GQ1b IgG antibody is associated with ophthalmoplegia in Miller Fisher syndrome and Guillain-Barré syndrome: clinical and immunohistochemical studies. Neurology 1993;43: 1911-17.

8 Liu JX, Willison HJ, Pedrosa-Domellöf F. Immunolocalization of GQ1b and related gangliosides in human extraocular neuromuscular junctions and muscle spindles. Invest Ophthalmol Vis Sci 2009;50:3226-32.

9 Koga M, Yoshino H, Morimatsu M, et al. Anti-GT1a IgG in Guillain-Barré syndrome. J Neurol Neurosurg Psychiatry 2002;72:767-71. 